University of Nebraska - Lincoln

DigitalCommons@University of Nebraska - Lincoln

$1-24-2008$

\title{
Synthesis and magnetic characterizations of manganite-based composite nanoparticles for biomedical applications
}

\author{
Aswini K. Pradhan \\ Center for Materials Research, Norfolk State University, apradhan@nsu.edu \\ R. Bah \\ Norfolk State University, Norfolk, Virginia \\ R. B. Konda \\ Norfolk State University, Norfolk, Virginia \\ R. Mundle \\ Norfolk State University, Norfolk, Virginia \\ H. Mustafa \\ Norfolk State University, Norfolk, Virginia \\ See next page for additional authors
}

Follow this and additional works at: https://digitalcommons.unl.edu/physicssellmyer

Part of the Physics Commons

Pradhan, Aswini K.; Bah, R.; Konda, R. B.; Mundle, R.; Mustafa, H.; Bamiduro, O.; Rakhimov, Rakhim R.; Wei, Xiaohui; and Sellmyer, David J., "Synthesis and magnetic characterizations of manganite-based composite nanoparticles for biomedical applications" (2008). David Sellmyer Publications. 208.

https://digitalcommons.unl.edu/physicssellmyer/208

This Article is brought to you for free and open access by the Research Papers in Physics and Astronomy at DigitalCommons@University of Nebraska - Lincoln. It has been accepted for inclusion in David Sellmyer Publications by an authorized administrator of DigitalCommons@University of Nebraska - Lincoln. 


\section{Authors}

Aswini K. Pradhan, R. Bah, R. B. Konda, R. Mundle, H. Mustafa, O. Bamiduro, Rakhim R. Rakhimov, Xiaohui Wei, and David J. Sellmyer 


\title{
Synthesis and magnetic characterizations of manganite-based composite nanoparticles for biomedical applications
}

\author{
A. K. Pradhan, ${ }^{\text {a) }}$ R. Bah, R. B. Konda, R. Mundle, H. Mustafa, O. Bamiduro, and \\ R. R. Rakhimov \\ Department of Engineering \& Center for Materials Research, Norfolk State University, 700 Park Avenue, \\ Norfolk, Virginia 23504, USA \\ Xiaohui Wei and D. J. Sellmyer \\ Department of Physics and Astronomy and Center for Materials Research and Analysis, \\ University of Nebraska, Lincoln, Nebraska 68588-0113, USA
}

(Presented on 8 November 2007; received 11 September 2007; accepted 5 October 2007; published online 24 January 2008)

\begin{abstract}
We report chemically synthesized highly crystalline lanthanum strontium manganite $\left(\mathrm{LaSrMnO}_{3}\right)$ and Eu-doped $\mathrm{Y}_{2} \mathrm{O}_{3}$ and their composites. The synthesis yields nanoparticles of size 30-40 nm. Magnetic measurements performed on nanoparticles and composites show magnetic transition at about $370 \mathrm{~K}$ with a superparamagnetic behavior at room temperature. The ferromagnetic resonance studies of the nanoparticles show large linewidth due to surface strains. The composite nanoparticles also display luminescent behavior when irradiated with ultraviolet light. The manganites as well their composite with the luminescent nanoparticles may be very useful for biomedical applications. (C) 2008 American Institute of Physics. [DOI: 10.1063/1.2829906]
\end{abstract}

\section{INTRODUCTION}

Magnetic nanoparticles of fairly uniform size and having a Curie temperature above room temperature are needed for most biomedical and magnetofluidic applications. Rare-earth based group of half-metallic ferromagnetic materials, such as manganites with a typical composition $\mathrm{La}_{0.7} \mathrm{Sr}_{0.3} \mathrm{MnO}_{3}$ (LSMO), are of interest in this context due to its high $T_{C}$ of $380 \mathrm{~K}$ and a large magnetic moment at room temperature. ${ }^{1-5}$ The half-metallic manganites are fairly metallic and can have large microwave absorption with the possibility of its use in hyperthermia applications and the large moment can also allow its use in marker experiments in biodetection ${ }^{6}$ at the same time. On the other hand, due to significant magnetoresistance effects in the immediate vicinity of the Curie point of manganites, eddy currents can be utilized as part of the heating mechanism. Hence, one can achieve the selective warming of the given areas of an organism by $T_{c}$-limiting production of controlled heat effects by means of an alternating external magnetic field. In general, particles that switch certain inherent properties "on/off" in relation to the relatively simple parameter, such as temperature, would provide possibilities for many potential designs of biomedical applications.

Another interesting material is $\mathrm{Eu}^{3+}$ ions, which show luminescent properties due to ${ }^{5} D_{0} \rightarrow{ }^{7} F_{2}$ transitions within and emits red light with a wavelength of $611 \mathrm{~nm}$, and can be used as the red phosphor. $\mathrm{Y}_{2} \mathrm{O}_{3}: \mathrm{Eu}^{3+}$ has a lumen equivalent brightness of $70 \%$ relative to $611 \mathrm{~nm}$ light and radiant efficiency of about $8.7 \%$ with better saturation without any detrimental effects. ${ }^{7,8}$ With the rapid advancement of biotechnology, nanoparticles of doped lanthanide oxides can be used

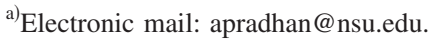

as promising labels because of their above mentioned optical properties, lack of photobleaching, and long luminescence lifetime $(\sim 1 \mathrm{~ms})$.

Coating the nanoparticles with a suitable material offers the possibility of attaching them to antibodies, proteins, medical drugs, etc. Therefore, studies on surface adsorption, the possibility of functionalizing and/or conjugating the particle coating with bioactive components, are also a crucial issue. There are only a few reports available for the synthesis of stochiometric manganite nanoparticles. ${ }^{9}{ }^{9}$ Here, we report on the synthesis and characterization of macromolecule encapsulated LSMO and Eu: $\mathrm{Y}_{2} \mathrm{O}_{3}$ composite nanoparticles.

\section{EXPERIMENTS}

$\mathrm{La}_{0.7} \mathrm{Sr}_{0.3} \mathrm{MnO}_{3}$ (hence called as LSMO) nanoparticles were synthesized by a sol-gel method from their acetate hydrate precursors, which were dissolved in water. This solution was mixed with citric acid solution in 1:1 volume ratio ultrasonically for about $30 \mathrm{~min}$. The mixture was heated in a water bath at $80{ }^{\circ} \mathrm{C}$ until all water is evaporated, yielding a yellowish transparent gel. The gel was further heated in an oven at $100{ }^{\circ} \mathrm{C}$ which formed a foamy precursor. This precursor decomposed to give black-colored flakes of extremely fine particle size on further heating at $400{ }^{\circ} \mathrm{C}$ for $4 \mathrm{~h}$. The flakes were ground and sintered at $800{ }^{\circ} \mathrm{C}$ for duration of $2 \mathrm{~h}$. Further heating in $\mathrm{O}_{2}$ ambient removed the carbon content. The nanopowders were coated with equal quantity of octadecyl amine (ODA) by magnetically stirring at $120^{\circ} \mathrm{C}$. A solution of ODA-coated LSMO and chloroform was prepared with a molarity of $10^{-2} \mathrm{M}$ and sonicated for $1 \mathrm{~h}$ for dispersion. The solution was rested for $24 \mathrm{~h}$ settling of the heavier LSMO particles. The solution containing suspended LSMO-ODA nanoparticles was decanted and purified using methanol several times in order to remove excess ODA from 


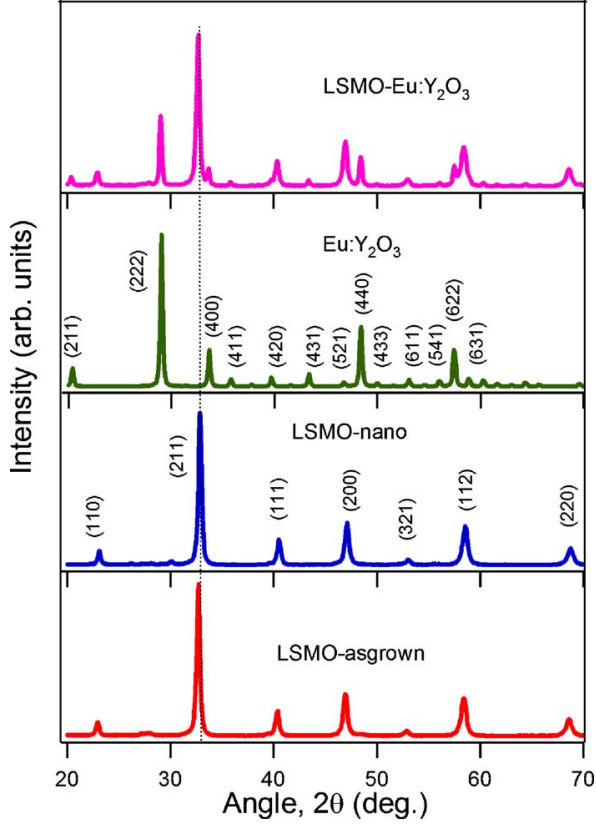

FIG. 1. (Color online) X-ray diffraction patterns of LSMO, EYO, and composite nanopowders.

the surface. The final purification was done using magnetic separation in order to remove any carbon content in the solution. The purified powder was naturally dried.

The nanocrystalline $\mathrm{Eu}^{3+}: \mathrm{Y}_{2} \mathrm{O}_{3}(\mathrm{EYO})$ powders were synthesized using a combustion technique ${ }^{11,12}$ from their respective nitrate solutions. LSMO and EYO nanopowders were thoroughly mixed and heated at $800{ }^{\circ} \mathrm{C}$ for $30 \mathrm{~min}$ to form a composite. These powders were coated with ODA and purified as described above.

\section{RESULTS AND DISCUSSION}

Figure 1 shows the powder x-ray diffraction (XRD) patterns of nanoparticle ensembles of manganites, doped rareearth oxides, and their composites. The purified manganite nanoparticles are highly crystalline and share the pseudocubic perovskite structure. However, the broadening of the XRD peaks is influenced by surface strain. Similar effects due to surface strains were observed in EYO nanoparticles. The XRD patterns of composite nanoparticles exhibit the characteristics of both LSMO and EYO nanoparticles.

Figures 2(a) and 2(b) show the representative fieldemission scanning electron microscope (FE-SEM) images and transmission electron microscope (TEM) image of LSMO and EYO nanoparticles, respectively. The size distribution is rather narrow, and the crystallite size is in the range of $30-40 \mathrm{~nm}$. Analysis of electron diffraction from nanoparticles and XRD patterns, such as those shown in Fig. 1, indicates that both manganite and EYO nanoparticles are highly crystalline and share the pseudocubic perovskite and cubic structures, respectively. Figures 2(c) and 2(d) show the FE-SEM images of LSMO and EYO composite nanoparticles, respectively, indicating distinctly well dispersed nanoparticles.

Figure 3 shows the temperature $(T)$ dependent magnetization $(M)$ of LSMO and composite nanoparticles in an ap-
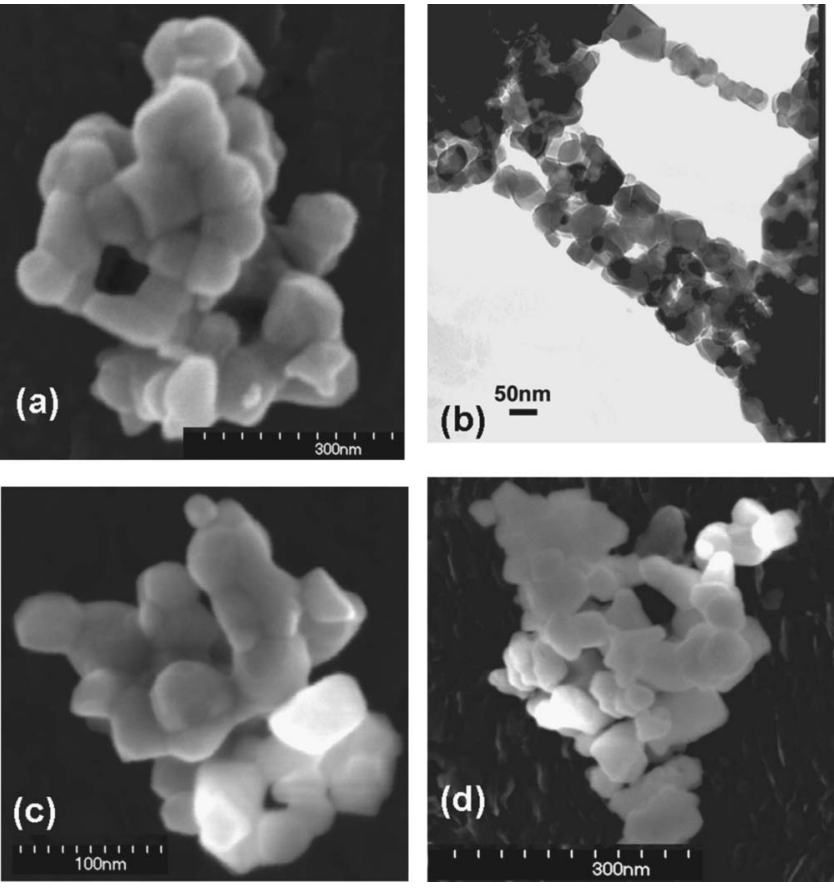

FIG. 2. (a) FE-SEM image of LSMO, (b) TEM image of Eu: $\mathrm{Y}_{2} \mathrm{O}_{3}$ nanopowders, and (c) and (d) are the FE-SEM image of LSMO and EYO composite nanopowders in two different scales.

plied magnetic field $(H)$ of $100 \mathrm{G}$. All samples show the onset of the magnetic transition at $370 \mathrm{~K}$ with a Curie temperature $\sim 360 \mathrm{~K}$. The as grown LSMO and LSMO+EYO composites show very similar magnetization behavior. The samples were cooled from $400 \mathrm{~K}$ down to $10 \mathrm{~K}$ under a magnetic field and then $M$ was recorded as the sample was warmed to $400 \mathrm{~K}$ under the same magnetic field [field cooled (FC)]. The results clearly illustrate the occurrence of ferromagnetic-to-paramagnetic phase transitions in all three samples as the temperature is increased. On the other hand, the coated nanoparticles showed reduced magnetization

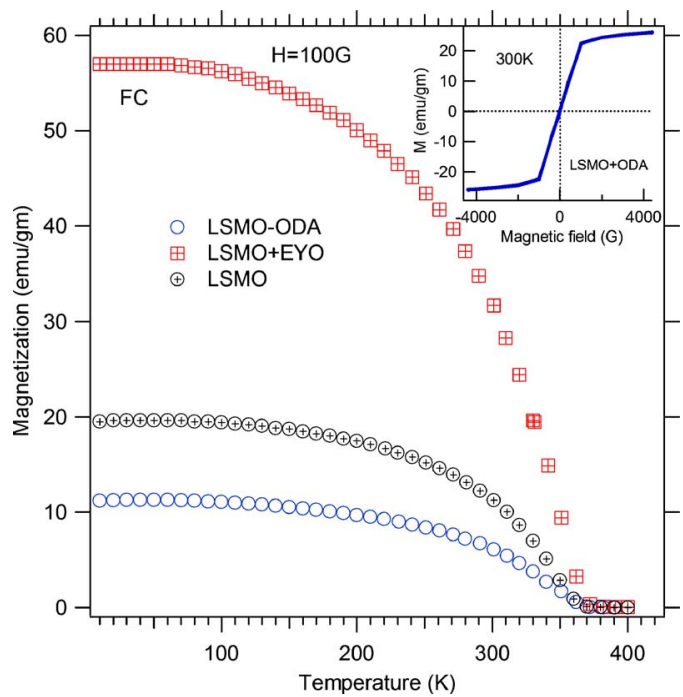

FIG. 3. (Color online) Temperature dependent field-cooled magnetization of as-synthesized LSMO, ODA-coated LSMO and composite (LSMO+EYO) nanopowders. The inset shows the magnetic hysteresis of LSMO nanoparticles. 


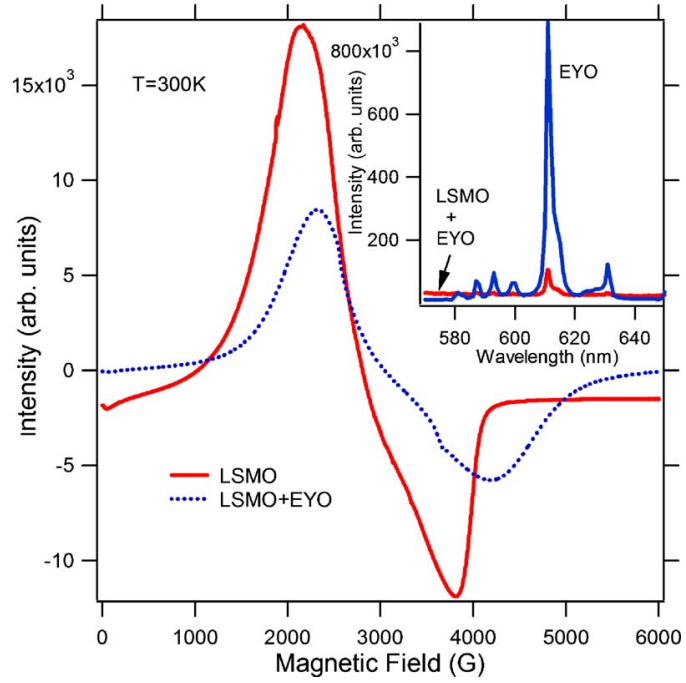

FIG. 4. (Color online) Ferromagnetic resonance spectra of LSMO and composite (LSMO+EYO) nanopowders. The inset shows the emission spectra of $\mathrm{Eu}: \mathrm{Y}_{2} \mathrm{O}_{3}$ and composite nanopowders.

compared to LSMO due to reduction in the volume fraction caused by ODA coating, as expected. The magnitude of the low temperature saturation magnetization also decreases with ODA coating, although hardly any reduction in the transition temperature was observed. Interestingly, the absolute magnetization increases in composite nanoparticles compared to that of LSMO. One of the reasons for such increase may be due to the local field enhancement at Eu site. However, further studies are necessary to resolve this issue. The slight reduction in transition temperature in these nanoparticles compared to their bulk counterpart $(\sim 375 \mathrm{~K})$ may be attributed to the surface-to-volume ratio in the nanoparticles, spin disorders at the surface, ${ }^{13}$ as well as due to the presence of superparamagnetic behavior of the nanoparticles. For nanoparticles of the order of tens of nanometers or less, one can see superparamagnetism, where the magnetic moment of the particle as a whole is free to fluctuate in response to thermal energy, while the individual atomic moments maintain their ordered state relative to each other, yielding anhysteretic, but sigmoidal $M-H$ curve. In fact, the superparamagnetic behavior is observed in these nanoparticles, and a magnetic field dependent magnetization curve is presented in the inset of Fig. 3 for LSMO nanoparticles.

Figure 4 shows the ferromagnetic resonance (FMR) spectra of LSMO and composite nanoparticles at $300 \mathrm{~K}$. FMR studies are probably the most sensitive method for detecting ferromagnetic order as well as the possible existence of other magnetic species. The narrower linewidth is generally taken as a signature of a homogeneous sample. However, the large FMR linewidth, which is in the range of $\sim 2 \mathrm{kG}$, indicates the effects of surface strains due to the size effect of the nanoparticles. No significant difference in the FMR curves was noticed, except a slight increase in resonance field in EYO nanoparticles.

The inset in Fig. 4 shows the emission spectra of EYO and the composite nanoparticles. Both spectra illustrate that the $\mathrm{Eu}^{3+}$ ions is in the cubic symmetry exhibiting the char- acteristics of red-luminescent $\mathrm{Y}_{2} \mathrm{O}_{3}: \mathrm{Eu}^{3+}$, in which ${ }^{5} D_{0}$ $\rightarrow{ }^{7} F_{2}$ transition at $\sim 610 \mathrm{~nm}$ is prominent, and the relatively weak emissions at the shorter wavelengths are due to the ${ }^{5} D_{0} \rightarrow{ }^{7} F_{1}$ transitions. However, the luminescence intensity in the composite nanopowders decreases significantly compared to EYO. The enhanced luminescence intensity in EYO can be attributed to the efficient energy transfer rate from the luminescence centers due to reduced interfaces. ${ }^{14}$ Therefore, the increased volume for the photon-solid interaction at the surface of $\mathrm{Y}_{2} \mathrm{O}_{3}: \mathrm{Eu}^{3+}$ nanopowder is one of the reasons for the enhanced emission intensity. However, the interface with LSMO in composites enhances the negative processes related to the surface effects.

\section{CONCLUSION}

We synthesized LSMO and macromolecule encapsulated LSMO and Eu: $\mathrm{Y}_{2} \mathrm{O}_{3}$ composite nanoparticles by the chemical routes. Both nanopowders show very good crystalline quality. The composite nanopowders of LSMO and $\mathrm{Eu}: \mathrm{Y}_{2} \mathrm{O}_{3}$ were synthesized by mixing and controlled heat treatment. The FE-SEM and TEM images of the individual nanoparticles and composite nanopowders show that the particles are in the range of $30-40 \mathrm{~nm}$. The LSMO as grown, ODAcoated, and composite nanoparticles show magnetic transition around $370 \mathrm{~K}$. The composite nanoparticles show superparamagnetic behavior at $300 \mathrm{~K}$. The FMR studies of the nanoparticles show large linewidth due to the surface strains. The composite nanoparticles also show emission characteristics, indicating possibility of imaging under UV radiation.

\section{ACKNOWLEDGMENTS}

This work is supported by NSF (RISE) project. Research at the University of Nebraska is supported by NSF-MRSEC, ONR, and CMRA.

${ }^{1}$ S. S. Davis, Trends Biotechnol. 15, 217 (1997).

${ }^{2}$ H. Y. Hwang, S. W. Cheong, N. P. Ong, and B. Batlogg, Phys. Rev. Lett. 77, 2041 (1996).

${ }^{3}$ A. Chainani, M. Mathew, and D. D. Sarma, Phys. Rev. B 47, 15397 (1993).

${ }^{4}$ Z. Trajanovic, C. Kwon, M. C. Robson, K. C. Kim, M. Rajaswari, S. E. Lofland, S. M. Bhagat, and D. Fork, Appl. Phys. Lett. 69, 1007 (1996).

${ }^{5}$ J. M. De Teresa, C. Marquina, D. Serrate, R. Fernandez-Pacheco, L. Morellon, P. A. Algarabel, and M. R. Ibarra, Int. J. Nanotechnol. 2, 3 (2005).

${ }^{6}$ A. Pankhurst, J. Connolly, S. K. Jones, and J. Dobson, J. Phys. D 36, R167 (2003).

${ }^{7}$ G. Blasse and B. C. Grabmaier, Luminescent Materials (Springer, Berlin, 1994).

${ }^{8}$ T. Hase, T. Kano, E. Nakazawa, and H. Yamamoto, in Advances in Electronics and Electron Physics, edited by P. W. Hawkes (Academic, New York, 1990), Vol. 79, p. 135.

${ }^{9}$ J. J. Urban, L. Ouyang, M.-H. Jo, D. S. Wang, and H. Park, Nano Lett. 4, 1547 (2004).

${ }^{10}$ V. Uskoković, A. Kočak, M. Drofenik, and M. Drofenik, Int. J. Appl. Ceram. Technol. 3, 134 (2006); R. Rajagopal, J. Mona, S. N. Kale, T. Bala, R. Pasricha, P. Poddar, M. Sastry, B. L. V. Prasad, D. C. Kundaliya, and S. B. Ogale, Appl. Phys. Lett. 89, 023107 (2006).

${ }^{11}$ E. Zych, Opt. Mater. (Amsterdam, Neth.) 16, 445 (2001).

${ }^{12}$ K. Zhang, A. K. Pradhan, G. B. Loutts, U. N. Roy, Y. Cui, and A. Burger, J. Opt. Soc. Am. B 21, 1804 (2004).

${ }^{13}$ A. J. Millis, Nature (London) 392, 147 (1998).

${ }^{14}$ E. Zych, D. Hreniak, and W. Strek, J. Alloys Compd. 341, 385 (2002). 